\title{
Structural stability of open vortex beams
}

Ruoyu Zeng, ${ }_{1}^{1}$ Qi Zhao, ${ }^{1,2}$ Yijie Shen, ${ }^{3}$ Yidong Liu, ${ }^{1}$ and Yuanjie Yang ${ }^{*, 1}$

${ }^{1)}$ School of Physics, University of Electronic Science and Technology of China,

Chengdu 611731, China

${ }^{2)}$ Southwest Institute of Technical Physics, Chengdu 610041, China

3) Optoelectronics Research Center and Center for Photonic Metamaterials, Univeristy

of Southampton, Southampton SO17 1BJ, United Kingdom

(*Electronic mail: dr.yang2003@uestc.edu.cn)

(Dated: 11 October 2021)

Normally, the intensity patterns of vortex beams are closed rings, such as Laguerre-Gaussian beams and BesselGaussian beams. Recent studies showed that the vortex beams with open ring structures, namely, open vortex beams (OVBs), have non-trivial orbital angular momentum (OAM) spectrums and optical forces, and can be employed in many applications such as metrology and quantum information. However, the structural stability of OVBs, including the topological charge (TC) conservation and the intensity invariance, has not been studied yet. Here, we theoretically propose a generalized model of OVBs, using both geometrical ray-like trajectories and coherent wave-packets, and the structural stability of OVBs is validated by the geometrical envelope of ray bundles. Moreover, we experimentally demonstrated that such OVBs can be generated by a partial fork-grating (PFG). Our study reveals the structural characteristics of OVBs, which lays a foundation for the OVB's potential applications in optical manipulation and optical metrology, etc.

An optical vortex beam, during its propagation, presents a phase circulation around the core where the field intensity vanishes. Ever since vortex beams were discovered to be associated with a well-defined intrinsic orbital angular momentum $(\mathrm{OAM})^{1}$, extensive studies on generation ${ }^{2,3}$, detection ${ }^{4,5}$ and manipulation ${ }^{6,7}$ of vortex beams have given possibilities to various emerging applications, spanning fields as wide as optical communication ${ }^{8}$, optical imaging ${ }^{9}$, optical metrology ${ }^{10}$, particle manipulation ${ }^{11}$ and quantum information ${ }^{12,13}$. In the past 20 years, the diversity and complexity of the vortex family have been extraordinarily expanded ${ }^{14}$. It was found that optical vortices are not limited to axially propagating singularities, but can exist and evolve in many forms, such as vortex knots $^{15}$, time-varying vortices ${ }^{16}$, spatiotemporal vortices ${ }^{17}$, coherence vortices ${ }^{18}$ and vector vortices ${ }^{19}$.

Meanwhile, in classical optics, monochromatic scalar light fields can be represented by both geometrical ray-like trajectories and coherent wave-packets. The connection between the ray and wave picture of light is given by the eikonal equation at the high-frequency limit ${ }^{20}$. Such a theoretical consistency is sometimes referred to as the ray-wave duality, which provides useful ideas and techniques for tailoring new types of structured laser beams, e.g., ray-optical Poincaré spheres for structured beams ${ }^{21-23}$, propagation-invariant light with shaped caustics ${ }^{24}$, SU(2) geometric modes generated from cavities $^{25}$ and holograms ${ }^{26}$, high-dimensional classically entangled light ${ }^{27}$, and new interpretations of self-accelerating beams ${ }^{28,29}$. Inevitably, vortex beams, as an essential class of structured light, can be analyzed in the framework of ray-wave duality. From the wave perspective, the degree of the phase circulation in the vortex wave field is given by an integer index called topological charge (TC). In terms of geometrical optics, a vortex beam is composed of bundles of resemble and rotational rays, which arise from specific orbits in the transverse plane ${ }^{30}$.

To date, most studies on vortex beams have focused on the elucidation of singular beams with cylindrical symmetry. However, when such symmetric structures are broken, light beams can present many exceptional physical properties, which are of interest to practical techniques. An example of this is the spin-dependent momentum splitting caused by the spin-orbit interaction in rotational symmetry breaking ${ }^{31}$. For another instance, a fractional optical vortex is distorted due to the phase discontinuity, leading to an external obstruction when it induces rotations to microscopic particles ${ }^{32}$. Besides, the vortex beam under perturbations, resulting to an open ring structure, was mentioned several times in both theoretical works and vortex-beam-related applications ${ }^{33-43}$. A vortex beam subjected to the sector aperture changes its OAM states ${ }^{33,34}$, enabling measurable quantum correlations and entanglement ${ }^{35}$. At the same time, the behaviors of the vortex beams generated by blocked optical elements were observed $^{36-38}$. Recently, the focal and optical trapping of a radially polarized vortex beam with broken axial symmetry were realized ${ }^{39}$, and the OAM spectrum of the truncated vortex beam was used to measure object parameters ${ }^{40}$. Morevover, methods for healing and measuring obstructed vortex beams were also proposed ${ }^{41-43}$. Despite the numerous optical effects presented above, some fundamental issues are not discussed in these publications: Is a partial light ring still a stably existed vortex? If so, how does one characterize the important properties (e.g., TC and phase profile) of such vortices?

In this letter, we focus on these unrevealed properties of open vortex beams (OVBs), i.e., the vortex structure and conservation of the TC. We start with the defination of the OVB, and then use a conceptually simple but effective method to construct OVBs based on the geometry of rotational light rays. The structure of a hybrid OVB, consisting of two pure OVBs with opposite TCs, is examined using the same method. Further, a good approximation of the ideal OVB can be generated via a partial fork-grating (PFG). Output beams at dif- 


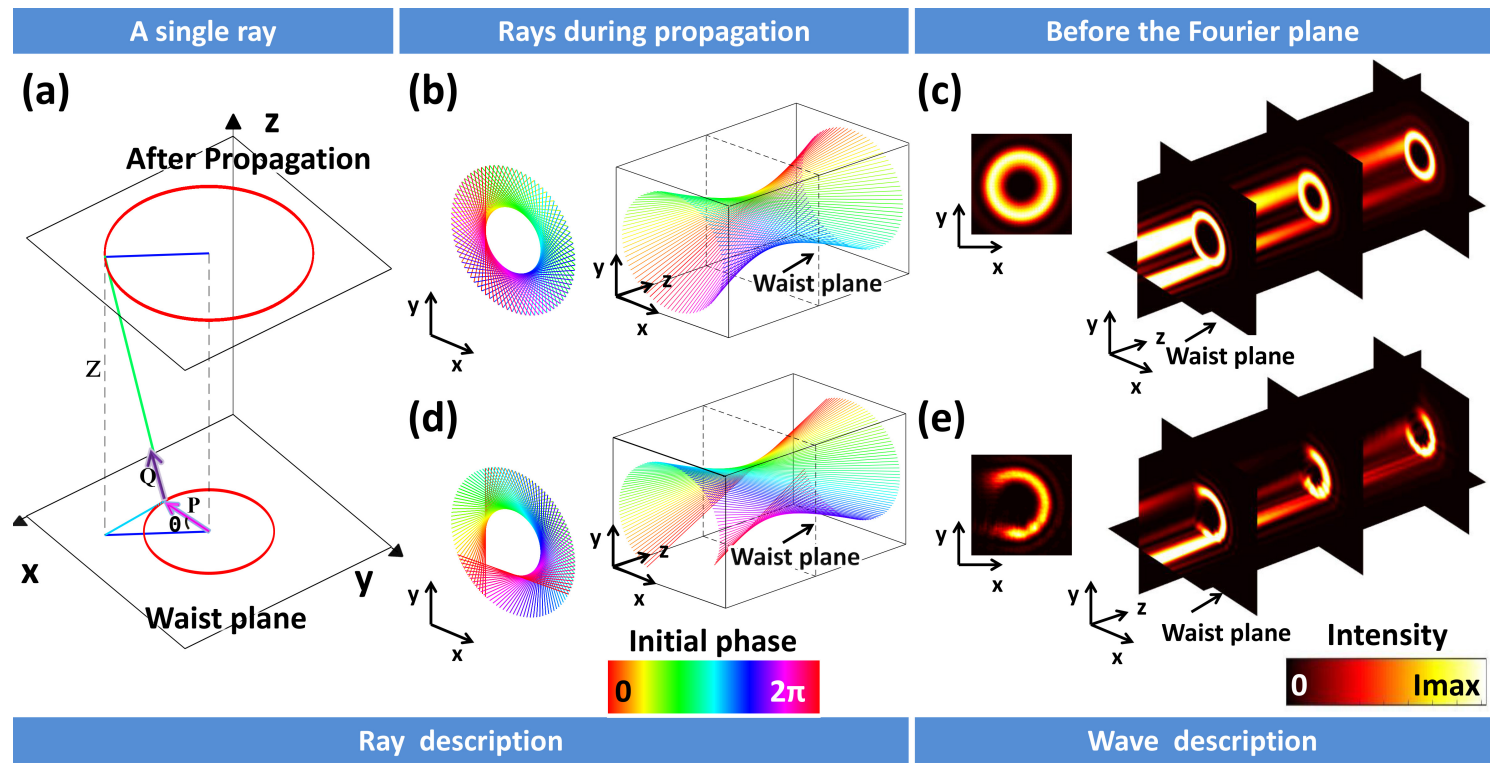

FIG. 1. (a): A single ray $\boldsymbol{r}$ with $\boldsymbol{P}, \boldsymbol{Q}$ being marked as well. (b),(c): The ray model (b) and wave field (c) of a circular vortex beam. (d), (e): The ray model (d) and wave field (e) of an OVB.The beams in (c),(e) are generated by fork-gratings and focused by a Fourier lens.

ferent diffraction orders are given by sums of hypergeometric modes. Thus, the OVBs are sketched through complementary wave and ray descriptions. Experimental corroborations which use PFGs and directly measure phase structures of OVBs are also carried out.

When a vortex beam propagates in free space, its structural stability ( or similarity) includes aspects such as the invariance of its vortex core, the conservation of the TC, and the resemble intensity pattern during its propagation. To study these properties of OVBs, we define an ideal OVB with the initial TC $l$ as a beam $E(\rho, \theta)$ with the transverse distribution of complex amplitude, which is given by:

$$
E(\rho, \theta)=A(\rho, \theta) \exp \left(i l_{0} \theta\right) \operatorname{rect}\left(\frac{\theta-\theta_{0} / 2}{\theta_{0}}\right)
$$

where $(\rho, \theta)$ are the polar coordinates, $A$ is the complex amplitude, $l_{0}$ is the change rate of the helical phase term, rect is the rectangle function ${ }^{44}$, and $\theta_{0}$ denotes the angular range of the partial ring. The TC of the initial field is calculated using results from Ref.[45]:

$$
l=l_{0} \times \frac{\theta_{0}}{2 \pi}
$$

This TC can be fractional or be an integer value. However, it is shown that the field after propagation should have an integer TC in order to be continuous ${ }^{46,47}$.

It is feasible to check the stability of an ideal OVB within the scope of ray optics. A propagating coherent monochromatic light field can be expressed as a family of parameterized rays. The stability of a light beam, is then converted into that of the overall geometrical shape of the rays. For twisted beams in free space, an element or a single ray $r$ in the beam is distinguished by two vectors $\boldsymbol{P}$ and $\boldsymbol{Q}$, which give the initial transverse location and direction of $\boldsymbol{r}$, respectively ${ }^{20}$. The ray $\boldsymbol{r}$ is expressed as

$$
\boldsymbol{r}=\boldsymbol{P}+\tau \boldsymbol{Q}
$$

where

$$
\left\{\begin{array}{l}
\boldsymbol{P}=\rho \hat{\boldsymbol{\rho}} ; \\
\boldsymbol{Q}=\frac{l_{0}}{k \rho} \hat{\boldsymbol{\theta}}+\hat{z}
\end{array}\right.
$$

with $\tau$ being the distance along $\boldsymbol{r}$ and $\hat{\boldsymbol{\theta}}, \hat{\boldsymbol{z}}$ being two of the base vectors in the cylindrical coordinates $(\rho, \theta, z)$. The ray $r$ is shown in Fig. 1(a), and a detailed derivation process can be found in Appendix A(See in supplementary materials).

One can sculpture a vortex beam with multiple arranged rays $r$ in Eq. (4). For a conventional circular vortex beam, it is useful to pick up a centered circle $\rho=\rho_{0}$ in the waist plane as a representative orbit of rays $r$, as outlined in Fig. 1(b). The envelope of rays determines the shape of the vortex beam. The corresponding wave field of this vortex beam is also shown in Fig. 1(c). To obtain the desired OVB in this article, we just need to have a partial orbit with a removed portion. Thus, the ray and wave picture of the OVB are illustrated in Fig. 1(d) and Fig. 1(e), respectively. In Fig. 1(d), the projection of all rays on every transverse plane $(x, y)$ is a geometrically-similar partial-ring envelope, which indicates that the beam's intensity shape is unchanged. At the meantime, one can assign a phase profile with an integer TC $l$ to the rays with an opening, which is color-labeled on Fig. 1(d). Consequently, an OVB with a selected TC as in Eq. (1) is formed. This colored phase profile is embedded in the configuration of rays. In each z-axial position, the phase difference of arbitrary two rays is a constant, so we can state that the phase circulation of an OVB is stable and the TC in Eq.(2) is conserved throughout the spreading process.

Also, an azimuthal rotation $\Delta \theta$ of $\boldsymbol{r}$ can be observed during the ray's extension as $z$ increases. Using the relation that the 
projection of this ray onto the waist plane is tangent to the circular orbit, $\Delta \theta$ is calculated as

$$
\Delta \theta=\arctan \left(\frac{l_{0} z}{k \rho^{2}}\right)
$$

When $z$ tends to the infinity, $|\Delta \theta|$ becomes $\pi / 2$. If $l>0$, $\Delta \theta=+\pi / 2$; if $l<0, \Delta \theta=-\pi / 2$. In Fig. 1(b), the same rotation rate is attached to each ray of an intact vortex beam. After propagation, any ray at an initial position $(\rho, \theta, 0)$ is replaced by another one from the same orbit, resulting to the invariance of the intensity. However, for the OVB in (d) and (e), the cylindrical symmetry breaks down and this spontaneous twist is exposed during propagation. Such a behavior looks like that of the so-called spiral beams ${ }^{48}$. Nevertheless, such a rotation does not affect the relative phase and amplitude distribution, leading to the conservation of TC.

The initial field of the OVB, after the certain optical path length, is mapped via the rays onto the other plane, which is analogous to the wave diffraction. To demonstrate this point, we use an example of hybrid OVBs. Two half-circumference OVBs with opposite TCs, separately placed in the left and right half space, produce a partial petal pattern near the Fourier plane (known as an azimuthal prism effect ${ }^{38}$ or a mirrorsymmetric OV used in optical manipulation ${ }^{49}$ ), as shown in Fig. 2(a). In the view of geometrical optics, the hybrid beam can be explained as two bundles of rays with opposite rotation rates, still located at the left and right respectively (Fig. 2(b)). The farther the beam travels, the greater the degree of the overlap between the two ray bundles, until they completely coincide and interfere in the far field. Of course, the number of the petals is an indirect evidence of the local phase distribution of an individual OVB.

In general, there can be two or more rays meeting at a same point, the amplitude mapping from some Cartesian coordinate points $\left(x_{j}, y_{j}, 0\right)$ is then written as in Ref.[50]:

$$
E(\boldsymbol{r})=C \sum_{j=1}^{N} E\left(x_{j}, y_{j}, 0\right) \exp \left(i k \int_{0}^{\tau_{j}} n^{2} d \tau_{j}\right)
$$

where $C$ is a constant relevant to the coordinate transformation between $(x, y, z)$ and $\left(x_{j}, y_{j}, \tau_{j}\right), N$ is the total number of

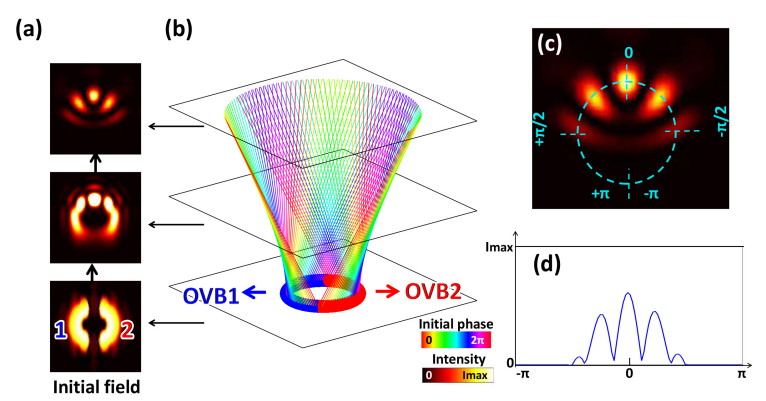

FIG. 2. (a): The simulated evolution of two OVBs $(l= \pm 2)$ residing at the left (OVB1, $l=-2$, blue) and right (OVB2, $l=+2$, red) space. (b): The equivalent two bundles of rays. (c):An orbit in the transverse petal pattern. (d): The estimated field intensity onto the orbit (c) using Eq.(6). rays at one point, $n$ is the refractive index of the medium, and $j$ is the indice of rays. Particularly, in a point of Fig. 2(b) at most two rays may come across. By Eq. (6), we can roughly estimate the intensity along an orbit (Fig. 2(c)) of the combined OVB from the initial field of Fig. 2(a), as can be seen in Fig. 2(d).

For an ideal OVB, its stability fully depends on the geometrical structure of rays. Although it is not possible to generate such an OVB with precisely tailored rays (Fig.1(d)), a favorable approximation of it can still be implemented based on diffraction devices, and this OVB still retains its stability to a certain extent, as the generated light field does not have intense distortions during its propagation. To demonstrate this, we have derived a wave-field representation of OVBs generated by modified fork-holograms.

Here, a partial fork-grating (PFG) is utilized. The transmission function of the PFG in the polar coordinates $(r, \varphi)$ is defined as:

$$
T(r, \varphi)=\sum_{m=-\infty}^{+\infty} t_{m} \exp \left[i m\left(l_{0} \varphi-\beta r \cos \varphi\right)\right] \operatorname{rect}\left(\frac{\varphi-\varphi_{0} / 2}{\varphi_{0}}\right)
$$

where $t_{m}$ is the transmission coefficient, $\beta=2 \pi / D$ with $D$ being the period of the grating. As the parameters of the PFG, $l_{0}$ is now the singularity index of the grating, and $\varphi_{0}$ denotes the angular range that light can pass through. In this case, an initial TC of $l$ in Eq.(2) is defined in the first-order diffracted beam.

Now using a Gaussian beam as the incident beam of the grating, the electric field at $z=0$ is written as

$$
E(r, \varphi, 0)=\exp \left(-\frac{r^{2}}{w^{2}}\right) T(r, \varphi)
$$

Here, $w$ is the waist width of the Gaussian beam. When this beam illuminates the PFG, two OVBs are formed in the +1 and -1 order of the diffraction pattern with opposite opening orientations, as outlined in Fig. 3(a). The simulated intensity and phase profile of the discrete vortex array are outlined in Fig. 3(b) and 3(c). With the paraxial propagation of the initial field in Eq. (8), its complex amplitude at distance $z$ can be decided. A thorough report regarding the propagation of the output pattern is put in Appendix $\mathbf{B}$ (See in supplementary materials). The OVBs in the Fresnel and Fraunhofer zone have the same form of expression. In a plane $(\rho, \theta)$ offset by the distance $z$ from the initial plane $(r, \varphi)$, as well as in the farfield regime, the OVB of the diffraction order $m$ is expressed as

$$
\begin{aligned}
& E_{m}(\rho, \theta, z)=\frac{-i k}{2 \pi z} \exp (i k z) \exp \left(i \frac{k}{2 z} \rho^{2}\right) \\
& \times \sum_{p=-\infty}^{+\infty} \pi a_{m p}\left(\frac{k \rho}{2 z}\right){ }^{|p|} w^{|p|+2} \frac{\Gamma\left(\frac{1}{2}|p|+1\right)}{\Gamma(|p|+1)} \\
& \times{ }_{1} F_{1}\left(\frac{1}{2}|p|+1,|p|+1 ;-\frac{k^{2} \rho^{2} w^{2}}{4 z^{2}}\right) \exp \left[i p\left(\theta \mp \frac{\pi}{2}\right)\right]
\end{aligned}
$$

where $\Gamma(x)$ is the Gamma function, ${ }_{1} F_{1}(a, b ; x)$ is a confluent hypergeometric function, and $p$ is the indice of hypergeomet- 


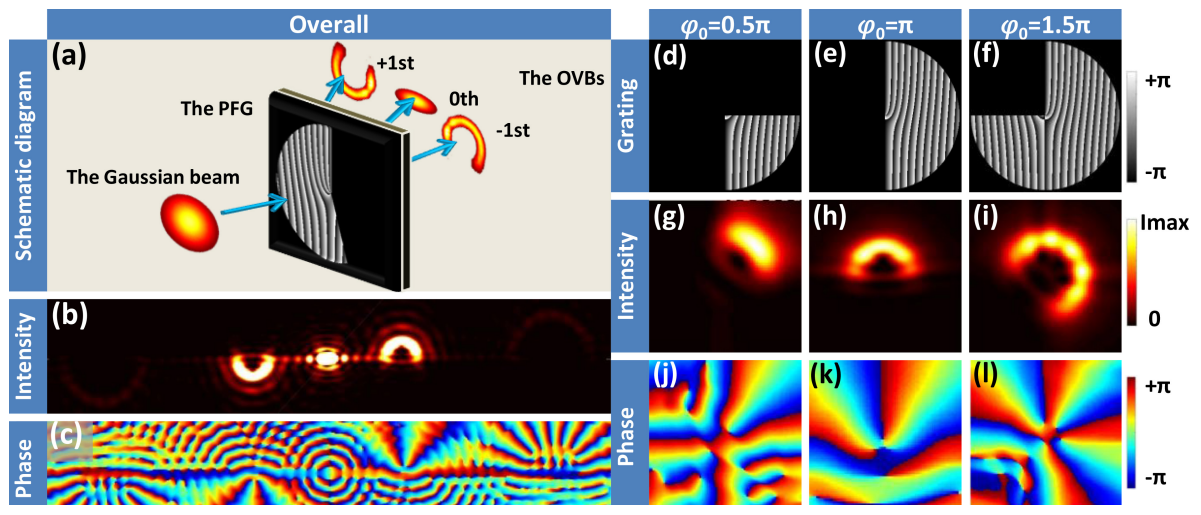

FIG. 3. (a) Schematic diagram of the OVB generated using a Gaussian beam diffracted by the PFG. (b)-(c): The output (b) intensity and (c) phase pattern at the Fourier plane. (d)-(f): PFGs with the parameters: $l_{0}=4$; (d) $\varphi_{0}=\pi / 2$, (e) $\varphi_{0}=\pi$, (f) $\varphi_{0}=3 \pi / 2$. (g)-(i): Far-field intensity distributions corresponding to (d)-(f). (j)-(l): Phase profiles corresponding to (g)-(i).

ric (HyG) vortex modes. The coefficients $a_{m p}$ are

$$
a_{m p}=\frac{\exp \left(\frac{i\left(m l_{0}-p\right) \varphi_{0}}{2}\right) \sin \left(\frac{\left(m l_{0}-p\right) \varphi_{0}}{2}\right)}{\pi\left(m l_{0}-p\right)}
$$

Meanwhile, the OVB in the near-field regime, has a very similar formula:

$$
\begin{aligned}
& E_{m}\left(\rho_{m}, \theta_{m}, z\right)=\frac{-i k}{2 z} \exp (i k z) \exp \left(i \frac{k}{2 z} \rho^{2}\right) \\
& \times \sum_{p=-\infty}^{+\infty} a_{m p} \frac{\rho_{m}^{|p|}}{2|p| \sqrt{\left(w^{-2}-i k / 2 z\right)^{|p|+2}}} \frac{\Gamma\left(\frac{1}{2}|p|+1\right)}{\Gamma(|p|+1)} \\
& \times{ }_{1} F_{1}\left[\frac{1}{2}|p|+1,|p|+1 ;-\rho_{m}^{2} /\left(4 w^{-2}-2 i k / z\right)\right] \exp \left[i p\left(\theta_{m}\right)\right]
\end{aligned}
$$

where

$$
\left\{\begin{array}{l}
\rho_{m}=\sqrt{m^{2} \beta^{2}+k^{2} \rho^{2} / z^{2}+2 m \beta k \rho \cos \theta / z} \\
\theta_{m}=\mp \arctan [\cot \theta+m \beta z /(k \rho \sin \theta)]
\end{array}\right.
$$

Note that the OVB is represented in both Eq. (9) and (11) as a sum of HyG modes instead of an entire analytical function, which indicates inhomogeneities in its cross-section. However, the same form of Eq. (9) and (11) makes sure that the overall appearance of the open vortex is similar to the original one. Specifically speaking, the total phase circulation of the beam remains stable, and the final TC is exactly what is defined by Eq. (2) and constrained by the parameters of the PFG, which will be shown later.

Also, each mode of Eq. (9) adds a $\pi / 2$ phase rotation, the direction of which depends on whether the energy flow of the OVB circulates in the clockwise or counterclockwise, namely, the sign of the TC. The open ring structure makes this rotational Gouy phase shift visible in the OVB's propagation.

An initial fractional charge of the field leads to the dynamic evolution of the TC and birth of local OVs in different regions of propagation ${ }^{46,47}$. For simplicity, we restrain the TC to take an integer value. Thus, the desired PFGs defined by Eq. (7) are shown in Fig. 3 (d)-(f). The essential parameters are $\varphi_{0}=$ $\pi / 2 \& \pi \& 3 \pi / 2$ and $l_{0}=4$. The far-field distributions of the OVBs generated by these gratings can be numerically fitted using Eq. (9), as shown in Fig. 3 (g)-(i). The OVBs have a quarter ring, a half ring and a three-quarter ring respectively. A stable rotating open ring before the beam's arrival at the Fourier plane can also be simulated using Eq. (11) from (As plotted in Fig. 1(e).).

What we are most concerned about is the stability of the vortex core: Whether the TC is conserved once the parameters of a PFG is determinate? To answer this, corresponding phase profiles are also illustrated in Fig. 3 (j)-(l). As can be seen, in the original angle range that light cannot be transmitted, the phase temporarily changes and then falls back, which does not contribute to the total phase ramp in a round trip. However, the local phase variation in the open ring remains stable. Thus, the vortex structure of the OVB is asymmetric and nonuniform, and this ultimate TC is the same as the initial TC $l$ of Eq. (2). This means that the TC is conserved during the beam's propagation. If the circumference of the open ring is picked properly, the phase increment in a cycle can be a multiple of $2 \pi$. The "cut-off" of the phase profiles in (j)-(l) results to TCs of 1,2 and 3. This indicates that an OVB can possess an arbitrary TC if $l_{0}$ and $\varphi_{0}$ are modulated.

To validate the theory and scheme stated above, we measure both the intensity and phase patterns diffracted by PFGs experimentally. The experimental setup used to generate and measure the OVBs is demonstrated in Fig. 4(a). A heliumneon laser with the wavelength of $632.8 \mathrm{~nm}$ emits a Gaussian beam. After the beam is collimated and broadened by a telescope system, it is diffracted by a computer-generated hologram $(\mathrm{CGH})$, which is equivalent to a PFG. An iris diaphragm is employed to select the +1 order diffracted beam, which finally can be observed in the focal range of a Fourier lens $L$ $(f=75 \mathrm{~cm})$.

Applying the above system, the $\pi / 2$ rotation of the OVB was probed by adjusting the position of the CCD within the focal length of $L$, as illustrated in Fig. 4(b). This rotation was not obvious when the beam initially passed $L$, but could be clearly recognized near the focal plane. The open ring was 
(a)

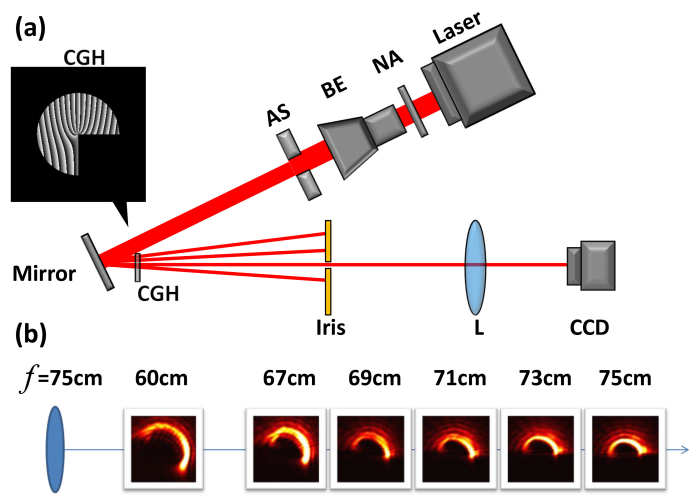

(c)

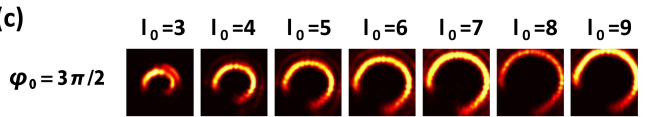

FIG. 4. (a) Schematic of the experimental set-up. NA, neutral attenuator; BE, beam expander; AS, aperture slot; $\mathrm{CGH}$, computergenerated hologram; CCD, charge-coupled device; $L$, a lens. (b) Experimental result of the OVB's rotation in the conditions of $l_{0}=$ $4, \varphi_{0}=\pi$. (c) Experimental result of the OVBs in the condition that $l_{0}=3 \sim 9$ and $\varphi_{0}=3 \pi / 2$ (all measured at the focal plane).

ultimately rotated by certain degrees compared with the input.

The intensity profiles recorded on the CCD are also shown in Fig. 4(c). We can see from the comparison that the experimental results are in high concordance with the fitting results according to the theory.

To directly measure the wavefront and TC of the OVB, a Shack-Hartmann wavefront sensor, consisting of a lenslet array and a position-sensing detector, was used to record the phase profile of the beam. The local phase aberration of one pixel from the OVB's wavefront is turned into an tilt from the centroid of the detector. Using data of these discrete tilts of all pixels, we can reconstruct the complex distributions along the partial rings. Fig. 5 (a1)-(a4) give four examples of measured phase distributions with specific grating parameters. Measured TCs are (a1):2.11; (a2): 3.06; (a3):3.70; (a4):3.85. Particularly, the cases of Fig. 5(a1) and (a2) are what we have encountered in simulations in Fig.2 and Fig.3. The TCs of different OVBs were calculated via phase circulations. For fractional OVBs, the effects of local vortices in the low intensity are omitted, so the total phase variations of these beams are not necessarily integers. Therefore, both experimentally and theoretically, we have confirmed that the actual TC is conserved once it is decided by the parameter of the PFG, which is also a straight evidence to the structural stability. With such a scheme, the relationship between the total phase increment and the parameters of the PFG were obtained, as manifested in Fig. 5 (b1)-(b2). We can see that the experimental results are in good agreement with the theory, which shows that the OVB can possess both an integer TC and an open ring structure.

In summary, we demonstrated that the reported OVB is a quasi-stable asymmetric vortex with an arbitrary integer TC, and its model is given by either the superposition of $\mathrm{HyG}$ wave modes or some tailored ray bundles. Our experimental results are in high concordance with the theoretical predictions. Our study provides a theoretical foundation for fundamental and applications of OVBs. Besides the optical force provided by the OVBs can be used for the guidance of microscopic particles with intended rotations and obstructions, the open ring structures and the phase circulations of OVBs are completely different from those of integer and fractional vortices, which is of importance to applications such as optical communications, optical metrology, and quantum information.
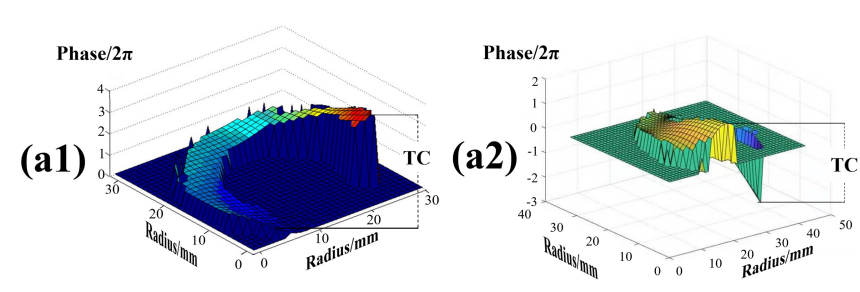

(b1)
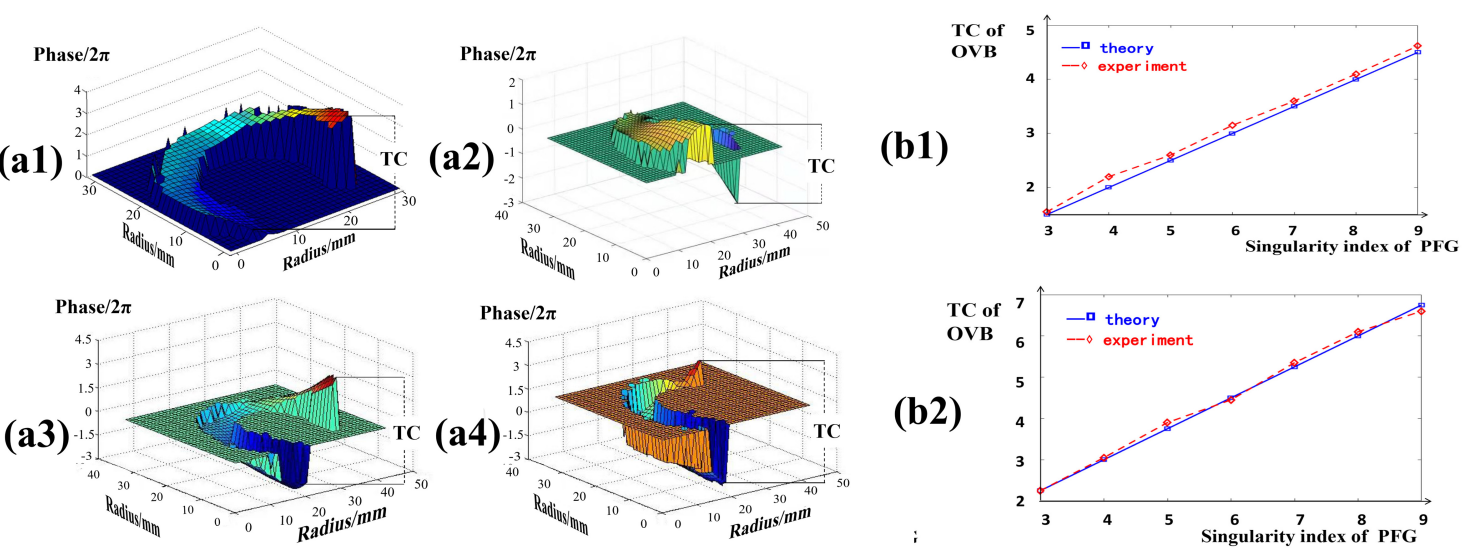

FIG. 5. (a1)-(a4): Experimentally probed wavefronts of OVBs generated using specific grating parameters: (a1) $l_{0}=4, \varphi_{0}=\pi$; (a2) $l_{0}=$ 4, $\varphi_{0}=3 \pi / 2$; (a3) $l_{0}=7, \varphi_{0}=\pi$; (b) $l_{0}=5, \varphi_{0}=3 \pi / 2$. (b1)-(b2): The experimental relationship between the TC and the singularity index of the PFG with (b1) $\varphi_{0}=\pi$, (b2) $\varphi_{0}=3 \pi / 2$.

\section{SUPPLEMENTARY MATERIAL}

derivations of the ray-wave model of OVBs.

See Supplementary Material for appendix of detailed 


\section{AUTHORS' CONTRIBUTIONS}

R.Zeng and Q.Zhao contributed equally to this aritcle. Y.Shen gave important suggestions in the ray model of the OVBs. Y.Liu helped with mathematical derivations. Y.Yang conceived the initial idea and supervised the whole project.

\section{ACKNOWLEDGEMENT}

This work is supported by The National Natural Science Foundation of China (11874102, 12174047), Sichuan Province Science and Technology Support Program (2020JDRC0006), and Fundamental Research Funds for the Central Universities under grant no. ZYGX2019J102.

\section{DATA AVAILABILITY}

The data that support the findings of this study are available from the corresponding author upon reasonable request.

${ }^{1}$ L. Allen, M. W. Beijersbergen, R. J. C. Spreeuw, and J. P. Woerdman, "Orbital angular momentum of light and the transformation of laguerregaussian laser modes," Phys. Rev. A 45, 8185-8189 (1992).

${ }^{2}$ N. R. Heckenberg, R. McDuff, C. P. Smith, and A. G. White, "Generation of optical phase singularities by computer-generated holograms," Opt. Lett. 17, 221-223 (1992).

${ }^{3}$ V. V. Kotlyar, A. A. Almazov, S. N. Khonina, V. A. Soifer, H. Elfstrom, and J. Turunen, "Generation of phase singularity through diffracting a plane or gaussian beam by a spiral phase plate," J. Opt. Soc. Am. A 22, 849-861 (2005).

${ }^{4}$ G. C. G. Berkhout and M. W. Beijersbergen, "Method for probing the orbital angular momentum of optical vortices in electromagnetic waves from astronomical objects," Phys. Rev. Lett. 101, 100801 (2008).

${ }^{5}$ Q. Zhao, M. Dong, Y. Bai, and Y. Yang, "Measuring high orbital angular momentum of vortex beams with an improved multipoint interferometer," Photon. Res. 8, 745-749 (2020).

${ }^{6}$ Y. Yang, Q. Zhao, L. Liu, Y. Liu, C. Rosales-Guzmán, and C.-w. Qiu, "Manipulation of orbital-angular-momentum spectrum using pinhole plates," Phys. Rev. Applied 12, 064007 (2019).

${ }^{7}$ Y. Wen, I. Chremmos, Y. Chen, Y. Zhang, and S. Yu, "Arbitrary multiplication and division of the orbital angular momentum of light," Phys. Rev. Lett. 124, 213901 (2020).

${ }^{8} \mathrm{~J}$. Wang, "Advances in communications using optical vortices," Photon. Res. 4, B14-B28 (2016).

${ }^{9}$ L. Torner, J. P. Torres, and S. Carrasco, "Digital spiral imaging," Opt. Express 13, 873-881 (2005).

${ }^{10}$ V. D'Ambrosio, N. Spagnolo, L. Del Re, S. Slussarenko, Y. Li, L. C. Kwek, L. Marrucci, S. P. Walborn, L. Aolita, and F. Sciarrino, "Photonic polarization gears for ultra-sensitive angular measurements," Nat. Commun. 4, 2432 (2013).

${ }^{11}$ Y. Yang, Y. Ren, M. Chen, Y. Arita, and C. Rosales-Guzmán, "Optical trapping with structured light: a review," Adv. Photon. 3 (2021), 10.1117/1.AP.3.3.034001.

${ }^{12}$ A. Mair, A. Vaziri, G. Weihs, and A. Zeilinger, "Entanglement of the orbital angular momentum states of photons," Nature 412, 313-316 (2001).

${ }^{13}$ D. Cozzolino, E. Polino, M. Valeri, G. Carvacho, D. Bacco, N. Spagnolo, L. K. K. Oxenløwe, and F. Sciarrino, "Air-core fiber distribution of hybrid vector vortex-polarization entangled states," Adv. Photon. 1, 1 - 9 (2019).

${ }^{14}$ Y. Shen, X. Wang, Z. Xie, C. Min, X. Fu, Q. Liu, M. Gong, and X. Yuan, "Optical vortices 30 years on: Oam manipulation from topological charge to multiple singularities," Light Sci. Appl. 8, 1-29 (2019).

${ }^{15}$ J. Leach, M. R. Dennis, J. Courtial, and M. J. Padgett, "Vortex knots in light," New J. Phys. 7, 55-55 (2005).
${ }^{16}$ L. Rego, K. M. Dorney, N. J. Brooks, Q. L. Nguyen, C.-T. Liao, J. San Román, D. E. Couch, A. Liu, E. Pisanty, M. Lewenstein, L. Plaja, H. C. Kapteyn, M. M. Murnane, and C. Hernández-García, "Generation of extreme-ultraviolet beams with time-varying orbital angular momentum," Science 364 (2019), 10.1126/science.aaw9486.

${ }^{17}$ A. Chong, C. Wan, J. Chen, and Q. Zhan, "Generation of spatiotemporal optical vortices with controllable transverse orbital angular momentum," Nat. Photon. 14, 350-354 (2020).

${ }^{18}$ M. Dong, C. Zhao, Y. Cai, and Y. Yang, "Partially coherent vortex beams: Fundamentals and applications," Sci. China Phys. Mech 64, 224201 (2020).

${ }^{19}$ Y. Shen, X. Yang, D. Naidoo, X. Fu, and A. Forbes, "Structured ray-wave vector vortex beams in multiple degrees of freedom from a laser," Optica 7 , 820-831 (2020).

${ }^{20}$ M. Born, E. Wolf, A. B. Bhatia, P. C. Clemmow, D. Gabor, A. R. Stokes, A. M. Taylor, P. A. Wayman, and W. L. Wilcock, Principles of Optics: Electromagnetic Theory of Propagation, Interference and Diffraction of Light, 7th ed. (Cambridge University Press, 1999).

${ }^{21}$ M. A. Alonso and M. R. Dennis, "Ray-optical poincaré sphere for structured gaussian beams," Optica 4, 476-486 (2017).

${ }^{22}$ R. Gutiérrez-Cuevas, S. Wadood, A. Vamivakas, and M. Alonso, "Modal majorana sphere and hidden symmetries of structured-gaussian beams," Phys. Rev. Lett. 125, 123903 (2020).

${ }^{23}$ Y. Shen, Z. Wang, X. Fu, D. Naidoo, and A. Forbes, "Su (2) poincaré sphere: A generalized representation for multidimensional structured light," Phys. Rev. A 102, 031501 (2020).

${ }^{24}$ A. Zannotti, C. Denz, M. A. Alonso, and M. R. Dennis, "Shaping caustics into propagation-invariant light," Nat. Commun. 11, 3597 (2020).

${ }^{25}$ Y. Shen, X. Yang, X. Fu, and M. Gong, "Periodic-trajectory-controlled, coherent-state-phase-switched, and wavelength-tunable su(2) geometric modes in a frequency-degenerate resonator," Appl. Opt. 57, 9543-9549 (2018).

${ }^{26}$ Z. Wan, Z. Wang, X. Yang, Y. Shen, and X. Fu, "Digitally tailoring arbitrary structured light of generalized ray-wave duality," Opt. Express 28, 31043-31056 (2020).

${ }^{27}$ Y. Shen, I. Nape, X. Yang, X. Fu, M. Gong, D. Naidoo, and A. Forbes, "Creation and control of high-dimensional multi-partite classically entangled light," Light Sci. Appl. 10, 1-10 (2021).

${ }^{28}$ E. Espíndola-Ramos, G. Silva-Ortigoza, C. T. Sosa-Sánchez, I. JuliánMacías, A. González-Juárez, O. de Jesús Cabrera-Rosas, P. Ortega-Vidals, C. Rickenstorff-Parrao, and R. Silva-Ortigoza, "Classical characterization of quantum waves: comparison between the caustic and the zeros of the madelung-bohm potential," J. Opt. Soc. Am. A 38, 303-312 (2021).

${ }^{29}$ E. Espíndola-Ramos, G. Silva-Ortigoza, C. T. Sosa-Sánchez, I. JuliánMacías, O. de Jesús Cabrera-Rosas, P. Ortega-Vidals, A. González-Juárez, R. Silva-Ortigoza, M. P. Velázquez-Quesada, and G. F. T. del Castillo, "Paraxial optical fields whose intensity pattern skeletons are stable caustics," J. Opt. Soc. Am. A 36, 1820-1828 (2019).

${ }^{30}$ M. V. Berry and K. T. McDonald, "Exact and geometrical optics energy trajectories in twisted beams," J. Opt. A: Pure and Applied Optics 10, 035005 (2008).

${ }^{31}$ X. Ling, X. Zhou, K. Huang, Y. Liu, C.-W. Qiu, H. Luo, and S. Wen, "Recent advances in the spin hall effect of light," Rep. Prog. Phys. 80, 066401 (2017).

${ }^{32}$ S. H. Tao, X.-C. Yuan, J. Lin, X. Peng, and H. B. Niu, "Fractional optical vortex beam induced rotation of particles," Opt. Express 13, 7726-7731 (2005).

${ }^{33}$ S. Franke-Arnold, S. M. Barnett, E. Yao, J. Leach, J. Courtial, and M. Padgett, "Uncertainty principle for angular position and angular momentum," New J. Phys. 6, 103-103 (2004).

${ }^{34}$ A. Volyar, M. Bretsko, Y. Akimova, and Y. Egorov, "Orbital angular momentum and informational entropy in perturbed vortex beams," Opt. Lett. 44, 5687-5690 (2019).

${ }^{35}$ J. Leach, B. Jack, J. Romero, A. K. Jha, A. M. Yao, S. Franke-Arnold, D. G. Ireland, R. W. Boyd, S. M. Barnett, and M. J. Padgett, "Quantum correlations in optical angle-orbital angular momentum variables," Science 329, 662-665 (2010).

${ }^{36}$ J. Hamazaki, Y. Mineta, K. Oka, and R. Morita, "Direct observation of gouy phase shift in a propagating optical vortex," Opt. Express 14, 83828392 (2006). 
${ }^{37}$ D. M. Cottrell, J. A. Davis, and T. J. Hernandez, "Fraunhofer diffraction of a partially blocked spiral phase plate," Opt. Express 19, 12873-12878 (2011).

${ }^{38}$ J. A. Davis and J. B. Bentley, "Azimuthal prism effect with partially blocked vortex-producing lenses," Opt. Lett. 30, 3204-3206 (2005).

${ }^{39}$ Z. Man, L. Du, Y. Zhang, C. Min, S. Fu, and X. Yuan, "Focal and optical trapping behaviors of radially polarized vortex beam with broken axial symmetry," AIP Adv. 7, 065109 (2017).

${ }^{40}$ G. Xie, H. Song, Z. Zhao, G. Milione, Y. Ren, C. Liu, R. Zhang, C. Bao, L. Li, Z. Wang, K. Pang, D. Starodubov, B. Lynn, M. Tur, and A. E. Willner, "Using a complex optical orbital-angular-momentum spectrum to measure object parameters," Opt. Lett. 42, 4482-4485 (2017).

${ }^{41}$ S. Wei, D. Wang, J. Lin, and X. Yuan, "Demonstration of orbital angular momentum channel healing using a fabry-pérot cavity," Opto-Electronic Adv. 1, 180006 (2018).

${ }^{42}$ X. Li, L. Ma, J. Zeng, Z. Dong, L. Liu, F. Wang, B. J. Hoenders, Y. Cai, and X. Liu, "Determining the topological charge of an obstructed vortex beam via reconstructed phase distribution," Appl. Phys. Lett. 117, 251103 (2020).
${ }^{43}$ S. Qiu, Y. Ren, T. Liu, Z. Liu, C. Wang, Y. Ding, Q. Sha, and H. Wu, "Directly observing the skew angle of a poynting vector in an oam carrying beam via angular diffraction," Opt. Lett. 46, 3484-3487 (2021).

${ }^{44}$ D. Zwillinger, V. Moll, I. Gradshteyn, and I. Ryzhik, eds., Table of Integrals, Series, and Products (Eighth Edition), eighth edition ed. (Academic Press, Boston, 2014) pp. 637-775.

${ }^{45}$ V. V. Kotlyar, A. A. Kovalev, and A. V. Volyar, "Topological charge of a linear combination of optical vortices: topological competition," Opt. Express 28, 8266-8281 (2020).

${ }^{46}$ G. Gbur, "Fractional vortex hilbert's hotel," Optica 3, 222-225 (2016).

${ }^{47}$ V. V. Kotlyar, A. A. Kovalev, A. G. Nalimov, and A. P. Porfirev, "Evolution of an optical vortex with an initial fractional topological charge," Phys. Rev. A 102, 023516 (2020).

${ }^{48}$ V. Volostnikov, S. Kotova, and S. Kishkin, "Spiral light beams: characteristics and applications," Journal of Physics: Conference Series 536, 012001 (2014).

${ }^{49}$ X. Z. Li, H. X. Ma, H. Zhang, M. M. Tang, H. H. Li, J. Tang, and Y. S. Wang, "Is it possible to enlarge the trapping range of optical tweezers via a single beam?” Appl. Phys. Lett. 114, 081903 (2019).

${ }^{50}$ Y. A. Kravtsov and Y. I. Orlov, "Caustics, catastrophes, and wave fields," Soviet Physics Uspekhi 26, 1038-1058 (1983). 


\section{A single ray}

(a)

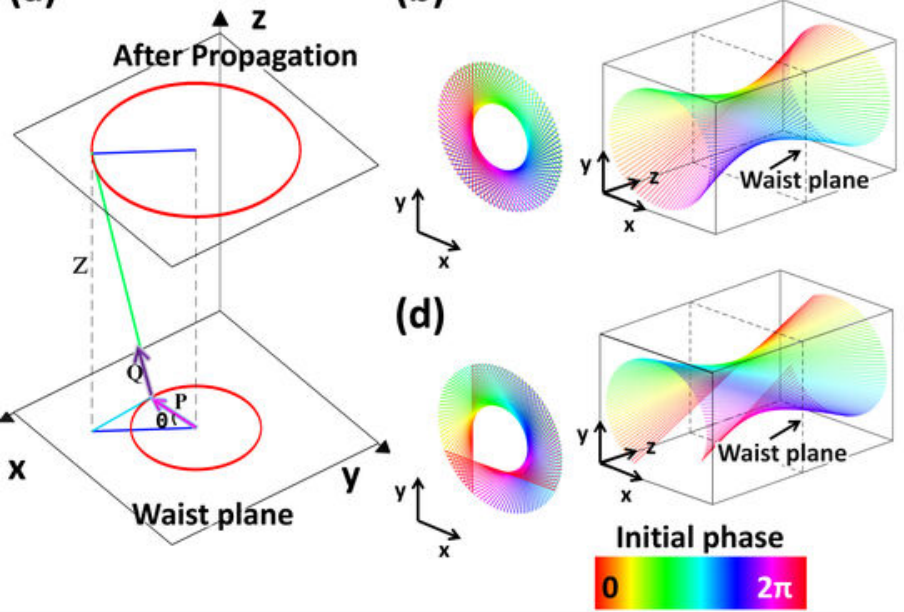

Ray description
Before the Fourier plane

(c)

(e)

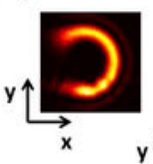

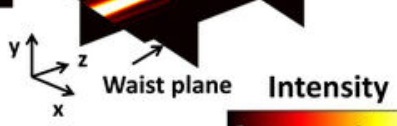

0

Imax

Wave description 
(a)

(b)
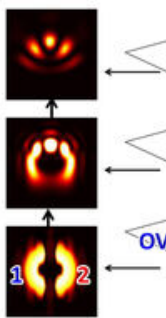

Initial field

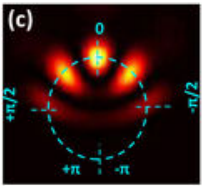

(d)

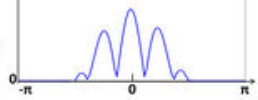

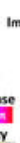

Intensity

Imax

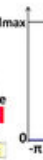




\section{Phase $/ 2 \pi$}

Phase $/ 2 \pi$

(a1)

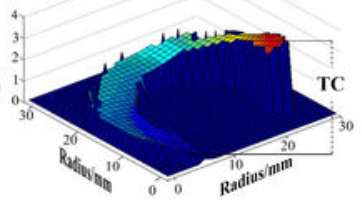

(a2)

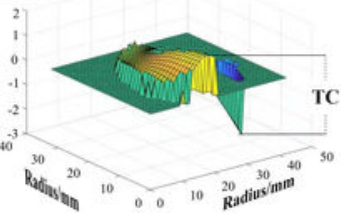

Phase $/ 2 \pi$

(a3)

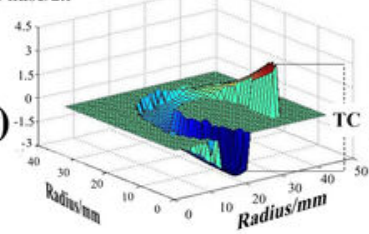

\section{Phase $/ 2 \pi$}

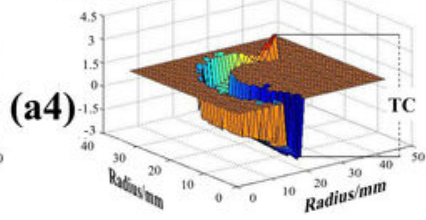

(b1)

$\begin{array}{ll}\begin{array}{l}\text { TC of } \\ \text { OVB }\end{array} & 5 \uparrow\end{array}$

${ }^{6} \stackrel{7}{7} \stackrel{8}{8}{ }^{9}$ Pingularity index of PFG

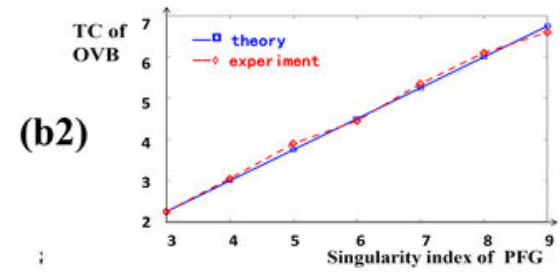

